\title{
Heart Chamber Volume at End Ventricular Diastole
}

National Cancer Institute

\section{Source}

National Cancer Institute. Heart Chamber Volume at End Ventricular Diastole. NCI

Thesaurus. Code C135372.

The volume of blood remaining in a heart chamber at end ventricular diastole. 